\title{
CT Score as a Mainstay of Tocilizumab Treatment in COVID-19
}

\author{
Vedrana Terkes ${ }^{1}$, Anela Tolic ${ }^{2}$, Miro Morovic ${ }^{* 1}$ \\ ${ }^{1}$ Department of Infectious Diseases, Zadar General Hospital, Zadar, Croatia \\ ${ }^{2}$ Department of Radiology, Zadar General Hospital, Zadar, Croatia \\ *Corresponding author: Prof. Miro Morovic, MD, PhD; Orcid Id: https://orcid.org/0000-0002-9745-074X \\ miro.morovic@gmail.com
}

Received 17 August 2021;

Accepted 08 September 2021;

Published 14 September 2021

\begin{abstract}
Background: After one year of numerous clinical trials, the position of tocilizumab in the treatment of COVOD-19 patients is not yet stable. Methods: In this small prospective observational study, we recruited patients with severe and critical COVID-19 with a rapid deterioration of their clinical status. Demographic, clinical, CT findings and laboratory data were collected prior to the decision to administer tocilizmab. Results: 33 patients were included between March 2020 and February 202111 (33.3\%) of patients died, with the median of 22.5 (9-35) days $(\mathrm{p}<0.05)$. Compared with the survivors, the patients who died were significantly older, with the mean age of 72.5 years vs 61.3 years, respectively. Also, the mean CT scores in the patients who died were significantly higher than in the survivors, with the mean value of 22.7 vs 17.3 ( $\mathrm{p}<0.05$ ). In addition, there was also a difference in the mean values of inflammatory parameters, which were generally higher in nonsurvivors. Conclusion: In this small exploratory analysis of 33 patients with severe or critical COVID-19, treated with tocilizumab we did not obtain neither a significant reduction of ICU admission neither of mortality. The high CT grade of lung damage has shown to be the only independent prognostic factor of clinical outcome, regardless of administration time or criteria of tocilizumab use.
\end{abstract}

Keywords: COVID-19, Tocilizumab, CT score, Clinical outcome

\section{Introduction}

Many studies that evaluated the use of tocilizumab for the treatment of COVID-19 gave inconclusive results and failed to demonstrate a reduction in the mortality rate ${ }^{[1-6]}$. Some recent studies showed a benefit of tocilizumab treatment in lowering the duration of ICU and hospital stays, and in reducing the rate of progression to mechanical ventilation or death; however, these studies did not also show improved survival ${ }^{[7,8]}$. Finally, after two very recent, large randomized controlled trials, Randomized, Embedded, Multi-factorial, Adaptive Platform Trial for Community-Acquired Pneumonia (REMAP-CAP) and Randomized evaluation of COVID-19 therapy (RECOVERY), which reported a mortality benefit of tocilizumab, the NIH guidelines have been changed and tocilizumab is recommended in certain patients with COVID-19 ${ }^{[9-11]}$.

In this prospective observational study, we aimed to present the treatment responses of tocilizumab in a small group of 33 severe or critically ill patients, which showed a rapid clinical deterioration with progressive oxygen demand, high inflammatory parameters and high CT scores.

\section{Methods}

Tocilizumab in patients with severe or critical COVID-19 disease is a observational study done in a tertiary care centre in Zadar County (169 853 inhabitants), Croatia (4,058 million inhabitants). The study population was adults ( $\geq 18$ years) with COVID-19, confirmed by PCR on nasopharyngeal swab, who were admitted to Zadar General Hospital between March 2020 and February 2021. On admission, all included patients had peripheral blood oxygen saturation $(\mathrm{SaO} 2)$ of less than $93 \%$ in room air and unilateral or bilateral X-ray lung infiltrates.

The patients were grouped according to the disease severity categorization ${ }^{[1,3]}$.

Initial evaluation included chest $\mathrm{x}$-ray, electrocardiogram (ECG), complete blood count (CBC) with differential and a metabolic profile, including liver and renal functional tests, Creactive protein (CRP), D-dimer, ferritin, interleukin-6 (IL-6) and procalcitonin; in almost all patients CT (computed tomography) was performed during the clinical deterioration and a semiquantitative CT severity scoring proposed by Pan et al was calculated ${ }^{[12]}$. 
The criteria for tocilizumab treatment were in accordance with the NIH Covid-19 treatment guidelines, i.e. tocilizumab was used in non-ICU patients who exhibited rapid clinical deterioration, with increasing oxygen needs and significant inflammatory response and in ICU patients who required invasive mechanical ventilation. Tocilizumab was administered at a daily dosage of $8 \mathrm{mg} / \mathrm{kg}$ ( $\max 800 \mathrm{mg}$ ) once, or rarely, the second dose (400 mg) was administered in three patients without adequate clinical response Dexamethason was given in every patient receiving tocilizumab, and in a number of them prior to the decision to include tocilizumab.

Antivirals, chloroquine and remdesivir were given generally on the admission of the patients.

Research was approved by the Ethics Committee of Zadar General Hospital. Since the patients were unable to give their informed consent, the Ethics Committees waived the requirement. All investigations were conducted according to the principles expressed in the Declaration of Helsinki.

\section{Results}

$33(5.6 \%)$ of 587 hospitalized patients during the investigational period received tocilizumab. The mean age was 65 years, and $91 \%$ were male. Most of the patients were severely $(11 ; 33.3 \%)$ or critically $(22 ; 63.6 \%)$ ill. In all the patients, the X-ray showed unilateral or bilateral pneumonia at admission; the mean CT score in $28(84.8 \%)$ of them was $18.9(10-25)$. Tocilizumab was given mostly during the second week $(21 ; 63.6 \%)$ from the symptoms onset, in $12(26.4 \%)$ of the patients during the third week. Only 3 patients received chloroquine phosphate while $17(51.5 \%)$ received remdesivir. All patients treated with tocilizumab received concomitantly dexamethasone, a number of them before tocilizuamb (Table 1).

$21(63.6 \%)$ patients were treated in ICU, where 9 of them received tocilizmab $(24,72.7 \%$ of patients received tocilizumab before ICU admission). Altogether, 11 (33.3\%) of the treated patients died, all during mechanical ventilation (Table 2). During the same period, the overall mortality in patients who did not receive tocilizumab was $18.4 \%$ (108/587).

The mean values of inflammatory parameters and CT score in tocilizumab treated patients are shown in Table 3. It must be pointed out that there was a high statistically significant difference in the parameters of age and CT score between the deceased and alive group of patients $(p<0.05)$. Also, the inflammatory parameters of CRP and IL- 6 were higher in nonsurvivors.

Table 1: Demographic and clinical characteristics of 33 COVID-19 patients treated with tocilizumab

\begin{tabular}{|l|l|}
\hline Age, median, range (years) & $65(26-95)$ \\
\hline Male $\mathrm{n}(\%)$ & $30(91)$ \\
\hline Comorbidities, $\mathrm{n}(\%)$ & $22(66.7)$ \\
\hline Hypertension & $13(39.4)$ \\
\hline Diabetes & $9(27.3)$ \\
\hline Hypertension and diabetes & \\
\hline Disease severity, $\mathrm{n}(\%)$ & $12(36.3)$ \\
\hline Severe & $21(63.6)$ \\
\hline Critical & \\
\hline Other medication used, n (\%) & $3(9)$ \\
\hline Chloroquine & $17(51.5)$ \\
\hline Remdesivir & \\
\hline Time of tocilizumab administration from first symptoms (weeks) n (\%) & $21(63.6)$ \\
\hline Second & $12(36.4)$ \\
\hline Third & \\
\hline Radiologic (X-ray, CT) findings of pneumonia, n (\%) & $33(100)$ \\
\hline X-ray & $28(84.8)$ \\
\hline CT & $18.9(10-25)$ \\
\hline CT-score, mean value (range) & \\
\hline
\end{tabular}

Table 2: Clinical course and outcome of our study population

\begin{tabular}{|l|l|}
\hline Non-survivors, $\mathrm{n}(\%)$ & $11(33.3)$ \\
\hline Survivors, $\mathrm{n}(\%)$ & $22(66.6)$ \\
\hline Hospital stay (days), median (range) & $19.4(9-42)$ \\
\hline Non-survivors & $22.5(9-35)$ \\
\hline Oxygenation, $\mathrm{n}(\%)$ & $4(12.1)$ \\
\hline Masks & $14(42.4)$ \\
\hline HFNO & $18(63.6)$ \\
\hline Mechanical ventilation & $21(63.6)$ \\
\hline ICU admission, $\mathrm{n}(\%)$ & \\
\hline Adverse events & $3(9.1)$ \\
\hline Leukopenia, $\mathrm{n}(\%)$ & \\
\hline
\end{tabular}

HFNO: High-flow nasal oxygen

ICU: Intensive care unit 
Table 3: Clinical, laboratory and radiologic characteristics in outcome analysis

\begin{tabular}{|l|l|l|l|}
\hline Variable & Non-survivors $(\mathbf{n = 1 1})$ & Survivors $(\mathbf{n = 2 2})$ & p-value* \\
\hline Age (years), median (range) & $72.5(60-91)$ & $61.3(25-95)$ & 0.0262 \\
\hline CRP (mg/L), median (range) & $162.5(99-302)$ & $171.4(66-302)$ & 0.7330 \\
\hline Ferritin (ng/ml), median (range) & $1292.2(100-2637)$ & $1596(618-7881)$ & 0.5352 \\
\hline Il-6 (ng/L), median (range) & $1145.4(25-6607)$ & $532.0(55-2998)$ & 0.2194 \\
\hline CT-score, median (range) & $22.7(18-25)$ & $17.3(12-25)$ & 0.0005 \\
\hline
\end{tabular}

*calculated using the Student's t-test

\section{Discussion}

After a year of numerous clinical and observational studies in the attempt to control the inflammatory response during the SARSCoV-2 infection, the last statement released recommends the use of tocilizumab in combination with dexamethason for hospitalized patients on supplemental oxygen for those who showed clinical progression and an increase in inflammatory parameters. However, the still remaining open questions are the identification of specific patients with respiratory deterioration who will benefit from this treatment, and also, adding tocilizmab to dexamethason in the case when there is no need for high-flow or noninvasive ventilation ${ }^{[11]}$.

Tocilizumab was administered in most of our patients $(21 / 63.6 \%)$ during the second week from the onset of the symptoms, in all after rapid clinical deterioration and increasing oxygen need; in 12 (36.4) of them it was given during the third week from the disease onset. $11(33.3 \%)$ of patients died; in survivors, the improvement (decrease of fever and oxygen need) ensued generally within 48-72 hours.

The main findings in this small exploratory analysis of 33 patients are: first, the fact that tocilizumab did not significantly reduce ICU admission and the risk of mechanical ventilation, since most of the patients who were transferred to the ICU $(21 / 63,6 \%)$ had earlier received tocilizumab; second, the administration of tocilizumab did not show mortality reduction since one third of the treated patients had died, and all of them among the critically ill patients on mechanical ventilation; third, the mean values of inflammatory parameters (CRP, ferritin, IL-6, D-dimer) in the deceased patients were much higher in comparison with those in the survivors; fourth, CT pulmonary damages were most pronounced in patients who had died, with the mean CT scores that were statistically significantly higher in comparison with those in survivors $(22.7: 17.3 ; \mathrm{p}<0.05)$.

The question of reducing ICU admission of COVID-19 patients is most frequently connected with the optimal timing of tocilizumab administration. Also, the difference in inflammatory responses between the group of non-survivors and survivors could be in part related to the timing of tocilizumab administration, but also to the possibility that cytokine dysregulation could be nonresponsive to immunomodulators in certain patients. Here, we must emphasized that the magnitude of inflammatory response must not correlate with the extent of lung damage and high mortality in COVID-19 ${ }^{[13]}$. Until presently, the question of optimal timing of tocilizumab use in COVID-19 has been extensively analyzed but has still not been resolved ${ }^{[4,14-16]}$. Moreover, one recent randomized, open label trial showed that tocilizumab was not superior to the standard of care in improving the clinical outcome in severe or critical COVID-19, and, moreover, that it might increase the mortality ${ }^{[17]}$.

The high mortality rate in our patients could be explained by the fact that fatal outcome was seen exclusively among the critically ill patients treated in the ICU. In addition, the mean age of non-survivors was statistically significantly higher than of alive patients $(72.5: 61.3$ years; $\mathrm{p}=0.0262)$.

It is well known that elderly individuals infected with COVID-19 are at greater risk of developing severe infection and complications, leading to increased morbidity and mortality rates $[18,19]$.

Finally, here it must be emphasized that the mean CT score in our patients was 18.9 , which means that most of the patients had already had severe lung damages when they received tocilizumab. These damages were most pronounced in nonsurvivors, with the mean CT score statistically significantly higher in comparison with the mean CT score in survivors (22.7:17.3; $\mathrm{p}<0.05)$; the predominant crazy-paving pattern was seen in nonsurvivors (Figure 1).

Since it has been shown that the CT score is in a high correlation with the clinical stages ${ }^{[20,12]}$, our finding of greater CT pulmonary severity in most of the patients who received tocilizumab during the second week from the disease onset was unexpected. Also, in our patients the mean CT score was in a good correlation with their laboratory findings (CRP, ferritin,IL-6, Ddimer).

It was earlier suggested that the CT score $\geq 18$ is highly predictive of the patient's mortality and that the CT parenchymal assessment may more accurately reflect a short-term outcome in comparison with non-specific inflammatory biomarkers ${ }^{[20,21]}$.

Others found that higher total CT scores (median $10 \mathrm{vs.}$ $4, \mathrm{p}<0.001$ ) were observed in the non-survivor group compared with the survivor group and that the crazy-paving pattern was more predominant in the non-survivor group than the survivor group $(39.0 \%$ vs. $12.0 \%, \mathrm{p}<0.001)$. It is also estimated that in the nonsurvivors, the total CT score rapidly increased over 10 points in the first 10 days ${ }^{[22]}$. Our findings are in complete agreement with these reports.

The key limitation of this study is the small sample size and, therefore, the insufficient power to detect assumed differences in clinical outcomes, in extent of CT damages and in inflammatory parameters related to the initiation of tocilizumab treatment in COVID-19. 


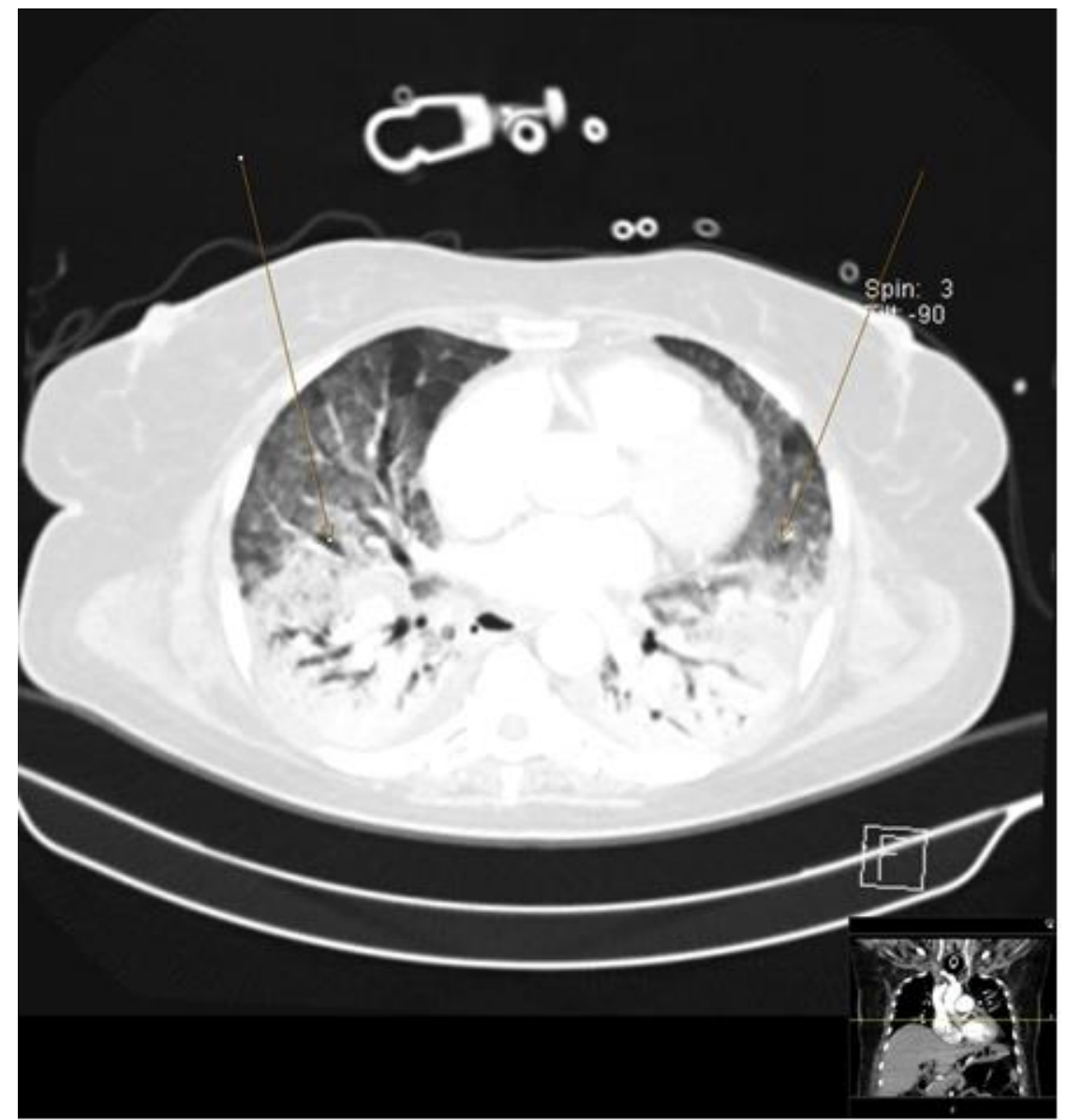

Chest CT-findings in elderly patient with a critical form of COVID-19 pneumonia on axial and coronal images; CT-score 25. A: 17th day of illness. Extensive bilateral lower lobes consolidation with air bronchogram and prominent vascular markings. Changes consistent with acute respiratory distress syndrome (ARDS).

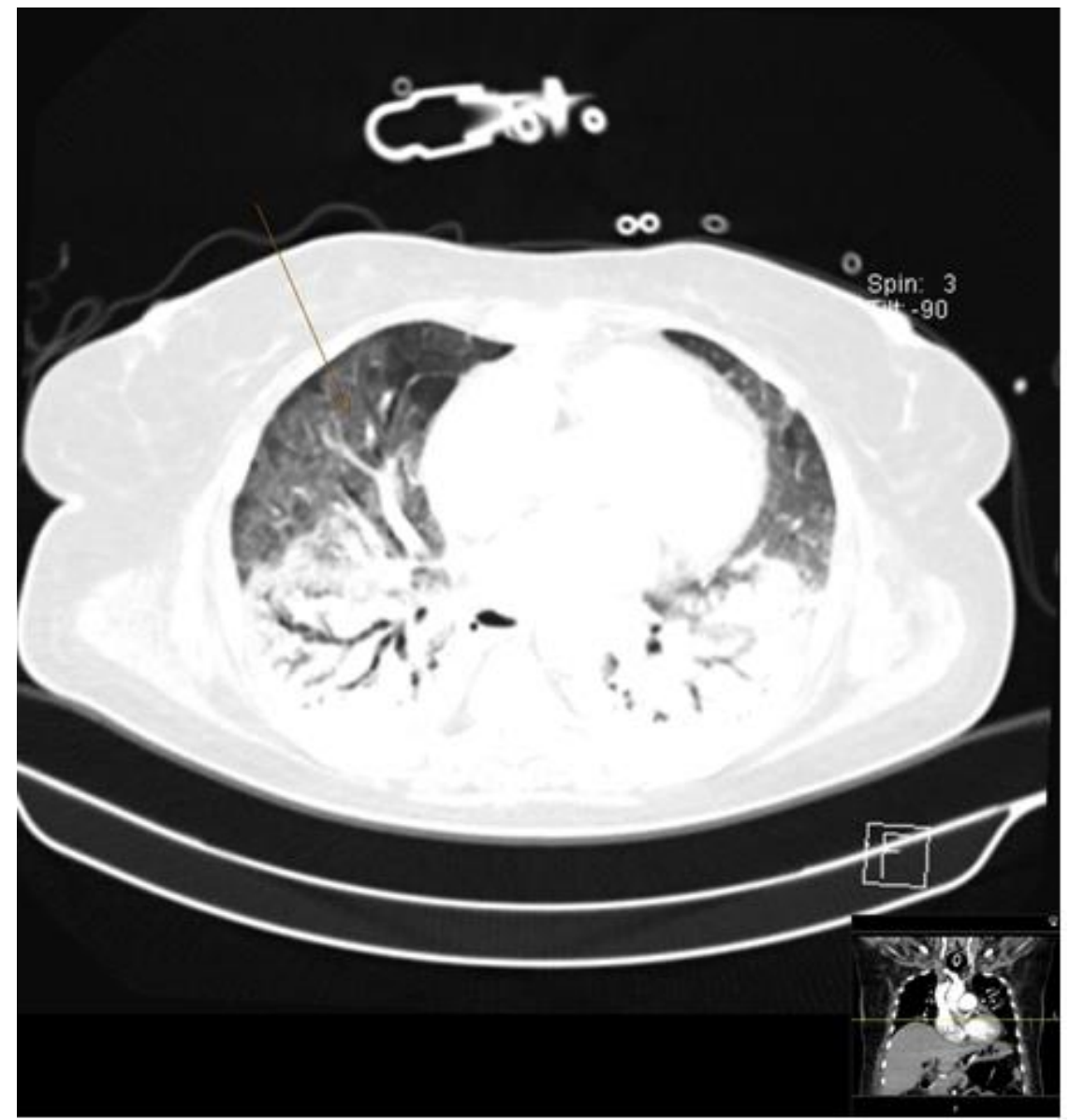

B: 17th day of illness, bilateral, crazy paving infiltrates, in the anterior parts of upper lobes. 


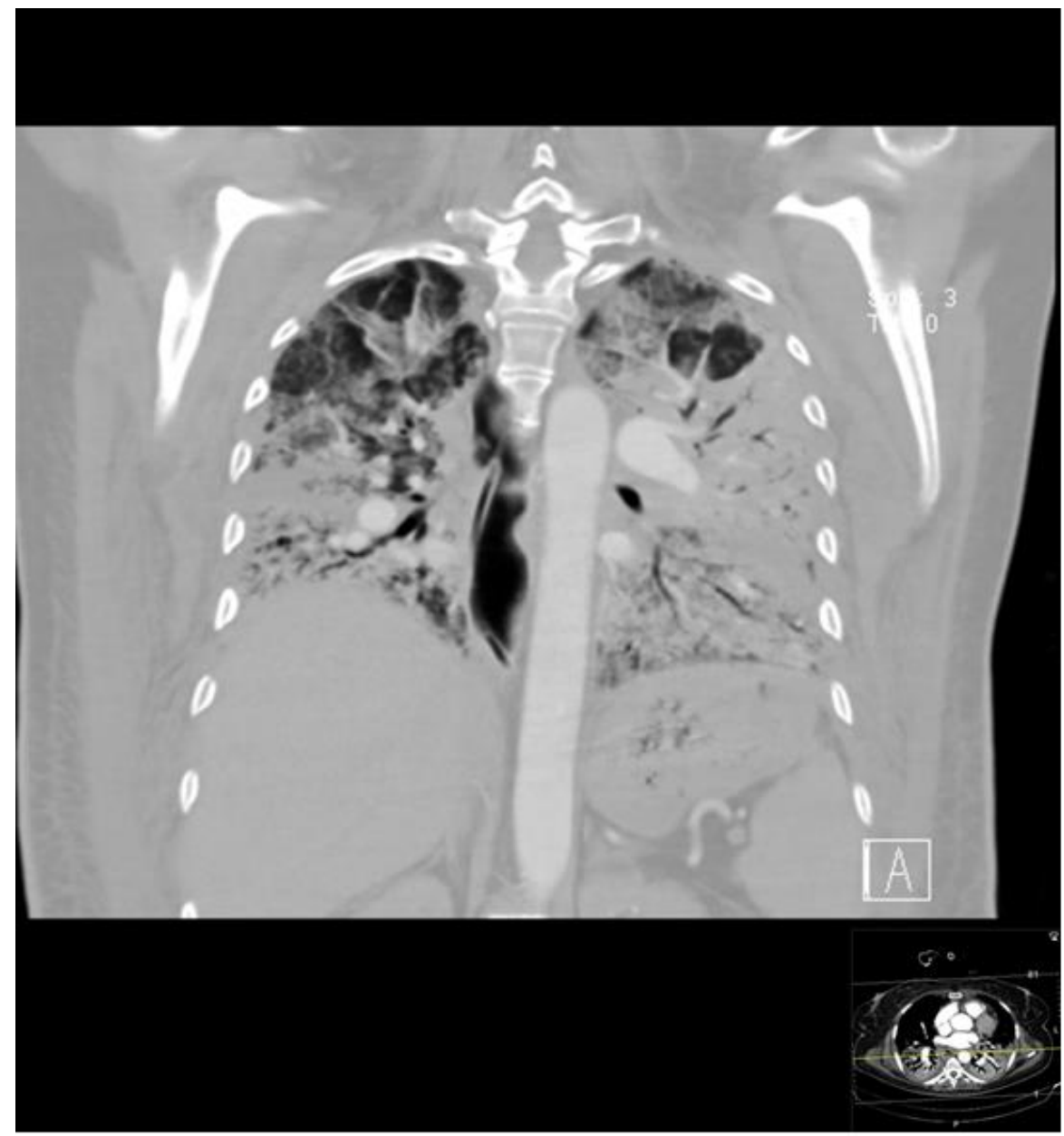

C: upper lobes crazy paving pattern and lower lobes consolidation

Figure 1: Chest CT-findings in elderly patient with a critical form of COVID-19 pneumonia on axial and coronal images; CT-score 25. A: 17th day of illness. Extensive bilateral lower lobes consolidation with air bronchogram and prominent vascular markings. Changes consistent with acute respiratory distress syndrome (ARDS). B: 17th day of illness, bilateral, crazy paving infiltrates, in the anterior parts of upper lobes. $\mathrm{C}$ : upper lobes crazy paving pattern and lower lobes consolidation

\section{Conclusion}

When summarized, in this small exploratory study of tocilizumab efficacy in COVID-19 patients, we have tried to homogenize the patient groups according to the disease severity, to the timing of treatment from the disease onset and to the extent of pulmonary CT damage and inflammatory parameters. Besides, tocilizumab was administered strictly according to the criteria of the recent treatment guidelines ${ }^{[11]}$, and taking into account the proposed 3stage classification system of COVID-19 with 3 grades of increasing severity which suggest the treatment approach ${ }^{[23,15]}$.

However, it has been confirmed yet again that the clinical course of COVID-19 is unpredictable, and that many factors could influence the course of the disease, the rapidity of pulmonary damage and development of complications and outcomes. Our results suggest that among these factors, in addition to the well known prognostic factors -age and adequate immunologic response-, the CT extent of pulmonary damage is probably the crucial factor of clinical (in) effectiveness of tocilizumab and outcome in patients, regardless of the time and criteria of its administration.

\section{Abbreviations}

COVID-19: Coronavirus disease 2019;

ICU: Intensive care unit;
CT: Computed tomography;

CRP: C-reactive protein;

IL-6: Interleukin 6;

HFNO: High-flow nasal oxygen.

\section{Conflicts of Interest}

The authors declare that there are no conflicts of interest regarding this paper.

\section{Funding Statement}

This work has not received any contribution, grant or scholarship.

\section{Authors' contributions}

All authors eaqully contributed to this paper.

\section{Confidentiality of Data}

The authors declare that they have followed the protocols of their work centre on the publication of data from patients.

\section{References}

[1] Stone JH, Frigault MJ, Serling-Boyd NJ, et al. BACC Bay Tocilizumab Trial Investigators. Efficacy of 
Tocilizumab in Patients Hospitalized with Covid-19. N Engl J Med. 2020 Dec 10;383(24):2333-2344.

[2] Hermine O, Mariette X, Tharaux PL, Resche-Rigon M, Porcher R, Ravaud P. CORIMUNO-19 Collaborative Group. Effect of Tocilizumab vs Usual Care in Adults Hospitalized With COVID-19 and Moderate or Severe Pneumonia: A Randomized Clinical Trial. JAMA Intern Med. 2021 Jan 1;181(1):32-40.

[3] Gupta S, Wang W, Hayek SS, et al. STOP-COVID Investigators. Association Between Early Treatment With Tocilizumab and Mortality Among Critically Ill Patients With COVID-19. JAMA Intern Med. 2021 Jan 1;181(1):41-51.

[4] Guaraldi G, Meschiari M, Cozzi-Lepri A, et al. Tocilizumab in patients with severe COVID-19: a retrospective cohort study. Lancet Rheumatol. 2020 Aug;2(8):e474-e484.

[5] Toniati P, Piva S, Cattalini M, et al. Tocilizumab for the treatment of severe COVID-19 pneumonia with hyperinflammatory syndrome and acute respiratory failure: A single center study of 100 patients in Brescia, Italy. Autoimmun Rev. 2020 Jul;19(7):102568.

[6] Jordan SC, Zakowski P, Tran HP, et al. Compassionate Use of Tocilizumab for Treatment of SARS-CoV-2 Pneumonia. Clin Infect Dis. 2020 Dec 15;71(12):31683173.

[7] Salama C, Han J, Yau L, et al. Tocilizumab in Patients Hospitalized with Covid-19 Pneumonia. N Engl J Med. 2021 Jan 7;384(1):20-30.

[8] Rosas IO, Bräu N, Waters $\mathrm{M}$, et al. Tocilizumab in Hospitalized Patients with Severe Covid-19 Pneumonia. N Engl J Med. 2021 Apr 22;384(16):1503-1516.

[9] REMAP-CAP Investigators, Gordon AC, Mouncey PR, Al-Beidh F, et al. Interleukin-6 Receptor Antagonists in Critically Ill Patients with Covid-19. N Engl J Med. 2021 Apr 22;384(16):1491-1502.

[10] RECOVERY Collaborative Group. Tocilizumab in patients admitted to hospital with COVID-19 (RECOVERY): a randomised, controlled, open-label, platform trial. Lancet. 2021 May 1;397(10285):16371645.

[11] COVID-19 Treatment Guidelines Panel. Coronavirus Disease 2019 (COVID-19) Treatment Guidelines. National Institutes of Health. Available at https://www.covid19treatmentguidelines.nih.gov/.

Accessed [May 24, 2021].
[12] Pan F, Ye T, Sun P, et al. Time Course of Lung Changes at Chest CT during Recovery from Coronavirus Disease 2019 (COVID-19). Radiology. 2020 Jun;295(3):715-721.

[13] Sinha P, Calfee CS, Cherian S, et al. Prevalence of phenotypes of acute respiratory distress syndrome in critically ill patients with COVID-19: a prospective observational study. Lancet Respir Med. 2020 Dec;8(12):1209-1218.

[14] Sinha P, Matthay MA, Calfee CS. Is a "Cytokine Storm" Relevant to COVID-19? JAMA Intern Med. 2020 Sep $1 ; 180(9): 1152-1154$. doi: 10.1001/jamainternmed.2020.3313

[15] de Simone G, Mancusi C. COVID-19: Timing is Important. Eur J Intern Med. 2020 Jul;77:134-135.

[16] Nugroho CW, Suryantoro SD, Yuliasih Y, et al. Optimal use of tocilizumab for severe and critical COVID-19: a systematic review and meta-analysis. F1000Res. 2021 Feb 4;10:73.

[17] Veiga VC, Prats JAGG, Farias DLC, et al. Coalition covid-19 Brazil VI Investigators. Effect of tocilizumab on clinical outcomes at 15 days in patients with severe or critical coronavirus disease 2019: randomised controlled trial. BMJ. 2021 Jan 20;372:n84.

[18] Triggle CR, Bansal D, Ding H,et al. A Comprehensive Review of Viral Characteristics, Transmission, Pathophysiology, Immune Response, and Management of SARS-CoV-2 and COVID-19 as a Basis for Controlling the Pandemic. Front Immunol. 2021 Feb 26;12:631139.

[19] Zhou F, Yu T, Du R, et al. Clinical course and risk factors for mortality of adult inpatients with COVID-19 in Wuhan, China: a retrospective cohort study. Lancet. 2020 Mar 28;395(10229):1054-1062.

[20] Francone M, Iafrate F, Masci GM, et al. Chest CT score in COVID-19 patients: correlation with disease severity and short-term prognosis. Eur Radiol. 2020 Dec;30(12):6808-6817.

[21] Colombi D, Bodini FC, Petrini M, et al. Well-aerated Lung on Admitting Chest CT to Predict Adverse Outcome in COVID-19 Pneumonia. Radiology. 2020 Aug;296(2):E86-E96.

[22] Pan F, Zheng C, Ye T, et al. Different computed tomography patterns of Coronavirus Disease 2019 (COVID-19) between survivors and non-survivors. Sci Rep. 2020 Jul 9;10(1):11336. 24. Siddiqi HK, Mehra MR. COVID-19 illness in native and immunosuppressed states: A clinical-therapeutic staging proposal. J Heart Lung Transplant. 2020 May;39(5):405-407. 